\title{
GRAND: A Giant Radio Array for Neutrino Detection
}

\author{
C. Timmermans ${ }^{1,2^{*}}$ for the GRAND collaboration
}

1 Nationaal Instituut voor Kernfysica en Hoge Energie Fysica (Nikhef), Science Park, Amsterdam, The Netherlands

2 Institute for Mathematics, Astrophysics and Particle Physics (IMAPP), Radboud

University, Nijmegen, The Netherlands

* c.timmermans@science.ru.nl

November 15, 2018

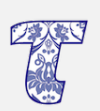

Proceedings for the 15th International Workshop on Tau Lepton Physics, Amsterdam, The Netherlands, 24-28 September 2018

scipost.org/SciPostPhysProc. Tau2018

\section{Abstract}

GRAND will be a distributed set of about 20 arrays of radio antennas, totalling $200,000 \mathrm{~km}^{2}$. We aim to study the ultra high energy universe through the detection of neutrinos, photons and cosmic rays at energies at the EeV scale. In addition GRAND will be used to study astronomical objects like FRBs and GRBs. We will describe the physics motivation, the detection strategy, the first stages of GRAND and the path to creating the complete setup.

\section{Contents}

\begin{tabular}{lll}
\hline & Introduction & 2
\end{tabular}

2 Physics Motivation 2

2.1 Cosmogenic neutrinos 2

2.2 Neutrinos from sources 3

2.3 GRAND as a photon detector 3

3 Detection Strategy 4

3.1 Radio detection of air showers 5

\begin{tabular}{|lll}
4 & The GRAND project & 5
\end{tabular}

4.1 GRANDProto35 6

\begin{tabular}{lll}
\hline 4.2 & GRANDProto300 & 6
\end{tabular}

$\begin{array}{lll}4.3 & \text { GRAND10K } & 7\end{array}$

\begin{tabular}{lll}
\hline 4.4 GRAND & 7
\end{tabular}

$\begin{array}{lll}5 & \text { Conclusion } & 7\end{array}$

\begin{tabular}{lr}
\hline References & 8
\end{tabular} 


\section{Introduction}

GRAND is an acronym for the Giant Radio Array for Neutrino Detection. GRAND is designed to allow for the detection of guaranteed cosmogenic neutrinos, even in pessimistic flux scenarios [1]. The energy of the cosmogenic neutrinos is in the EeV regime, three orders of magnitude above the astronomical neutrinos detected by the IceCube collaboration [2]. The expected flux level requires a new detection technique that allows to instrument a huge area at affordable costs. Recent developments and results of the radio detection of air showers [3] show that using radio antennas it is possible to measure the electromagnetic energy in air showers, the direction of the parent particle and the development of the air shower in the atmosphere with excellent precision. Even though the current generation radio-detection units are rather expensive, the GRAND collaboration constructed a roadmap to further develop the technique. This roadmap should lead to a cheaper detector with increased functionality, which will then allow for the construction of 10-20 arrays with a combined area of $200,000 \mathrm{~km}^{2}$. This note will detail the physics motivation as well as the roadmap developed by the GRAND collaboration.

\section{Physics Motivation}

The fundamental questions regarding the highest energy particles created in the Universe are:

- What are the sources of Ultra High Energy particles?

- What is the nature of Ultra High Energy Particles?

- What are the laws describing the interactions between Ultra High Energy Particles and matter?

The study of cosmic neutrinos with energies at the EeV level will impact all of these questions. As neutrinos are undeflected in space, they point back to their source. The flux of neutrinos is related to the composition of charged ultra high energy cosmic rays. Knowing the charged cosmic ray composition allows to study the laws governing the UHECR interactions with better precision. Furthermore, the neutrino-matter interactions themselves are sensitive to new physics.

\subsection{Cosmogenic neutrinos}

Cosmogenic neutrinos 4 originate from the interactions from ultra-high energy cosmic rays with the comic microwave background. The unknown parameters in the estimation of the flux on Earth are the cosmic ray composition at the highest energies and the astronomical evolution of the sources. In the left plot of Figure 1 the range of flux expectation is given together with the sensitivities of current and future experiments. Under the assumption that the flux of all $\nu$-generations on Earth is the same, the sensitivity of GRAND will be unrivalled. The expected flux-measurement using GRAND will provide a severe constraint on the amount of light nuclei in the UHECR-flux complementary to direct measurements of the composition by air shower experiments. It should be noted that the GRAND detector will also be used to measure cosmic ray induced air showers with an aperture that is an order of magnitude larger than the Pierre Auger Observatory, the largest cosmic ray detector to date. Therefore, the GRAND collaboration will be in a unique position to infer the cosmic ray composition both from direct measurements and indirectly through the neutrino flux. 

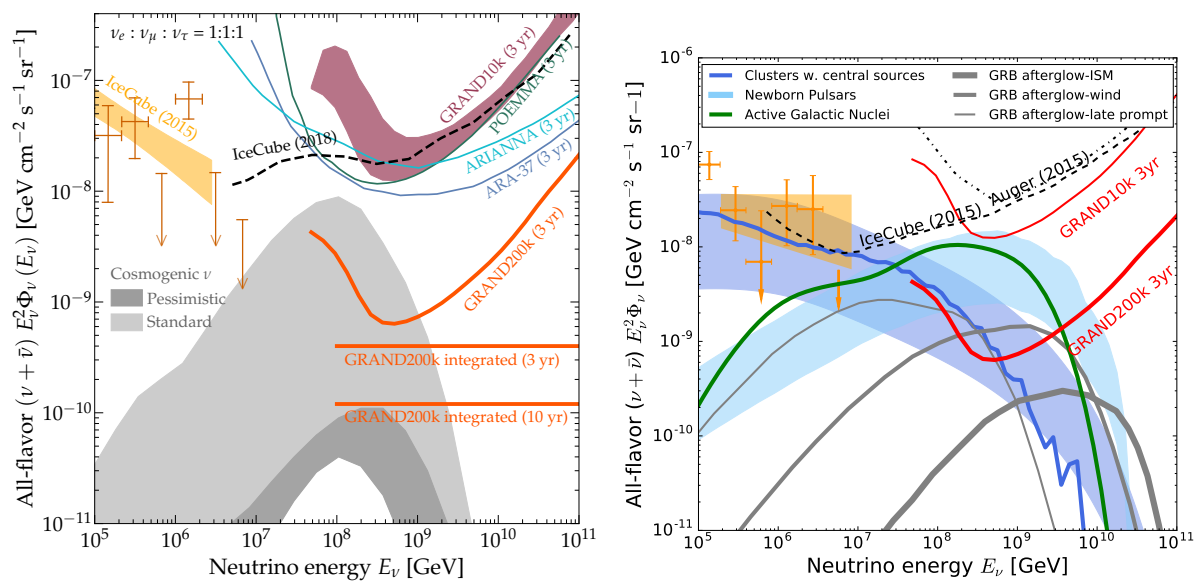

Figure 1: Left: Cosmogenic neutrino flux expectations [5] and (expected) sensitivities of current and future experiments [6-10]. Right: expected neutrino fluxes from sources using different source models $[11-15$.

\subsection{Neutrinos from sources}

In addition to cosmogenic neutrinos, it is expected that close to the sources of UHECR, the cosmic rays interact with matter by which neutrinos are created. The flux expectations depend on the nature of cosmic ray sources, as shown in the right plot of Figure 1. In many of the scenarios, the direct neutrino flux is larger than the cosmogenic flux. This, combined with the excellent angular resolution of better than $0.5^{\circ}$ that can be obtained using radio detection, will allow for a discovery of sources of ultra high energy cosmic rays in many scenarios, solving a century old question in physics.

\subsection{GRAND as a photon detector}
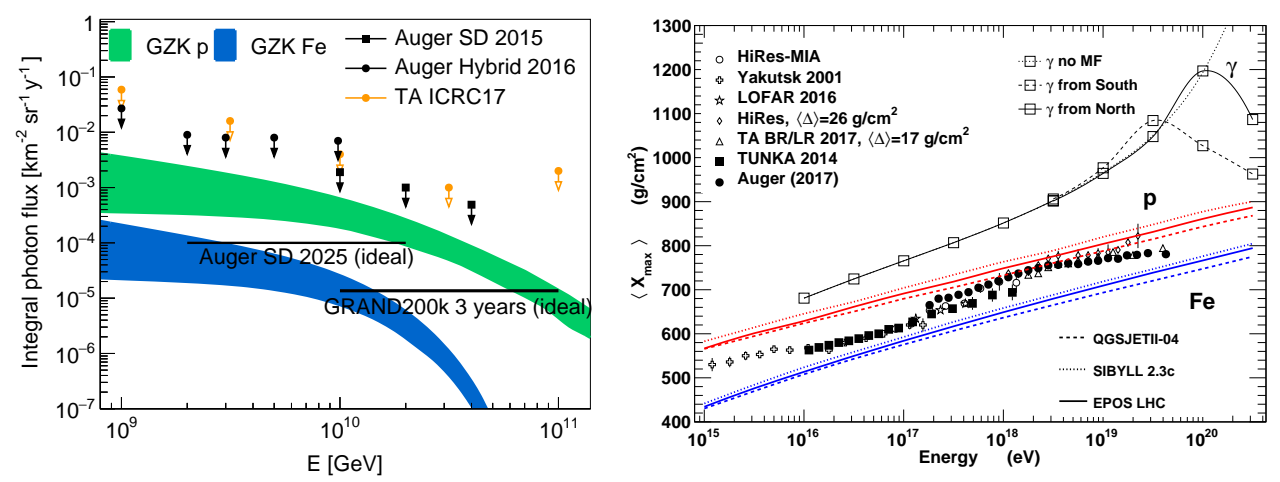

Figure 2: Left: GRAND sensitivity to UHE photons [16 19]. Right: Measurements of the average depth of shower maximum as a function of energy [20 26] as well as the results for several simulations 27 .

Similar to UHECR induced air showers, the GRAND detector is sensitive to photon induced air showers for photon energies beyond $10 \mathrm{EeV}$, as shown in the left part of Figure 2. The photon/hadron separation will be made based upon the depth of the shower maximum in the atmosphere (right part of Figure 2. The expected GRAND accuracy 
on this parameter is about $20 \mathrm{~g} / \mathrm{cm}^{2}$, whereas the difference between the average depth of shower maximum of photons and hadrons is more than $100 \mathrm{~g} / \mathrm{cm}^{2}$. The sensitivity to both neutrinos and photons at EeV energies will allow the GRAND collaboration to set stringent restrictions to the source evolution parameters of cosmic rays, thereby providing crucial information on the nature of sources of UHECR. If, in addition, sources are identified, the combined photon and neutrino measurement will provide new information on the amount of cosmic microwave background and infrared photons on the path between source and Earth.

As GRAND is a radio detector, it is also sensitive to radio signals from the universe with frequencies between 50 and $200 \mathrm{MHz}$. The background created by cosmic ray interactions in the galactic plane will be used to calibrate the antenna as this signal is known very well. In addition, flaring radio signals such as Giant Radio Pulses and Fast Radio Bursts will be searched for in this frequency domain. The GRAND measurements will lack directional information for these events, but due to the large number of antennas the GRAND measurement will be sensitive to the full sky overhead. Therefore GRAND will be the first detector to make a measurement on the number of these flaring signals

\section{Detection Strategy}

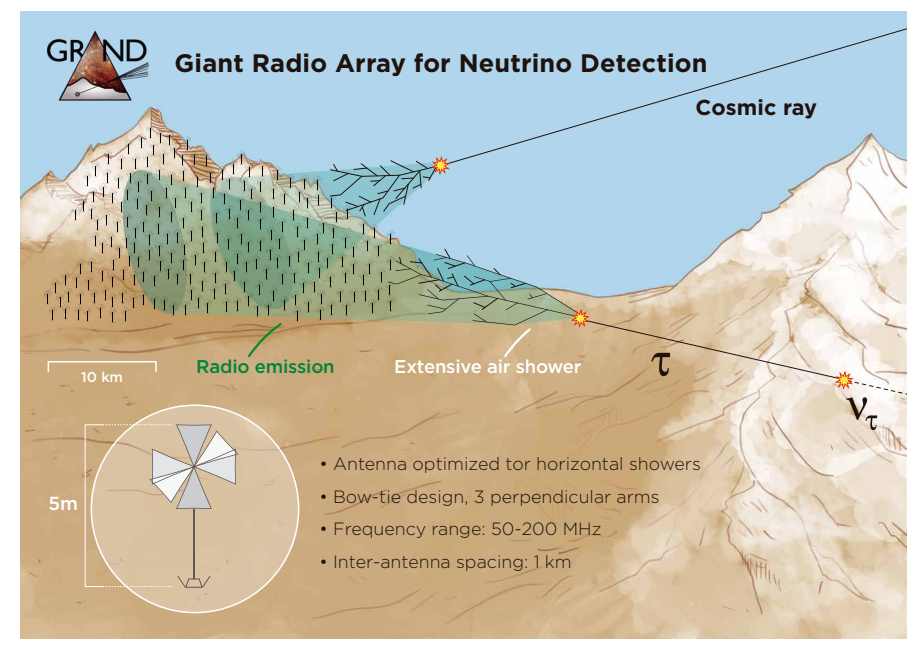

Figure 3: GRAND detection principle [1]

The GRAND detection strategy is outlined in Figure 3. The main neutrino channel the GRAND collaboration will be using is the detection of $\nu_{\tau}$ interactions in mountains from which a $\tau$-lepton emerges that decays in the atmosphere. The decay of the $\tau$ causes an air shower that will be detected by an array of radio antennas. Measurements in this channel are only possible due to neutrino oscillations, as mostly muon and electron neutrinos are created at the source or through the GZK-mechanism. This channel also enables the study of the $\nu_{\tau}$ cross section in rock and $\tau$-decay at Lorentz-factors of $10^{8}$, leading to decay lengths exceeding $50 \mathrm{~km}$.

In addition, cosmic rays and photons at ultra high energies will also cause air showers that can be detected by an array of radio antennas, thus allowing the GRAND collaboration to perform multi-messenger astro-particle physics at the highest energies. 


\subsection{Radio detection of air showers}

When an air shower develops in the atmosphere, the Earth magnetic field causes deflections of the fast number of electrons and positrons produced. The geo-magnetic radiation caused by this deflection is measurable in the $\mathrm{MHz}$ regime and is polarised in the direction of the Lorentz-force as shown in the left part of Figure 4. In addition, there is charge buildup at the front of the air shower, leaving positive ions behind in the atmosphere seen in the right part of Figure 4. This Askaryan effect causes a polarised emission, where the polarisation is directed towards the shower axis. The total radio signal is the vector-sum of both effects, and is thus not radial symmetric. The Cherenkov-effect, caused by the
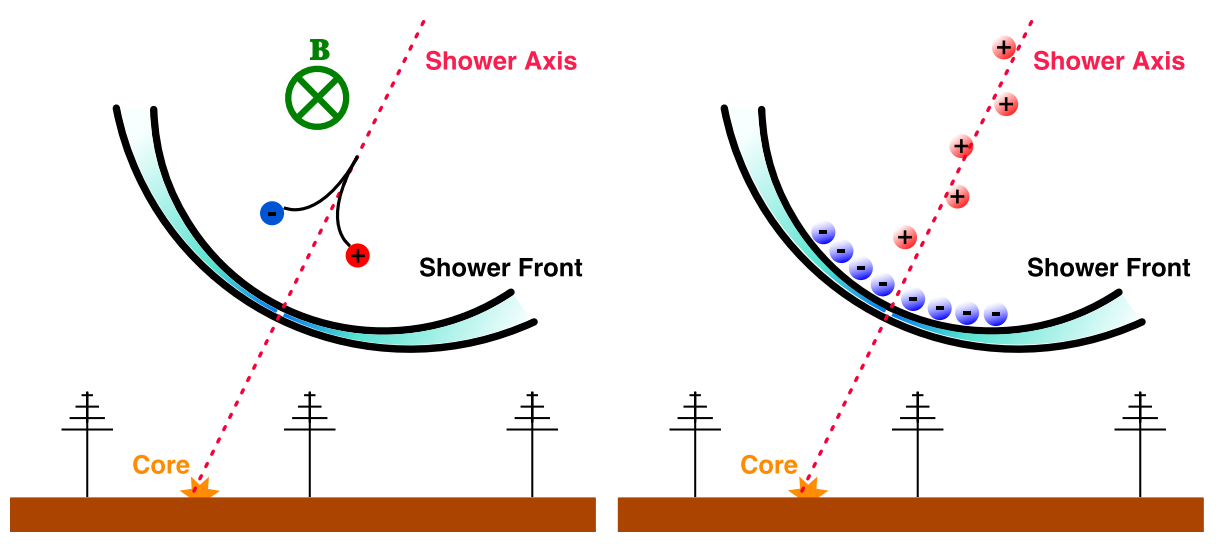

Figure 4: Schematic overview of radio emission mechanisms 28. Left: The geomagnetic mechanism. Right: The charge excess mechanism.

atmospheric index of refraction, provides a shower cone whose extend is a measure of the distance of the shower production to the measurement plane. The Cherenkov-cone is more apparent at higher frequencies, whereas the power of the signal is largest at lower frequencies. Therefore the GRAND design includes a wide-band measurement of the radio signal between 50 and $200 \mathrm{MHz}$. This frequency range is optimises the signal-to-noise ratio in conditions where there are only natural background sources. Man made backgrounds are either wide-band or single-frequency. The latter is easily removed through filtering at the input whereas the site-selection takes the amount of wide-band RFI explicitly into account. The trigger algorithms developed at the detector use the known signal shape of radio emission from air showers to distinguish the air shower signal from man-made noise. A higher level trigger combines the data from all stations. From the timing information both direction and distance of the source can be obtained. This will be used to remove remaining false triggers from the data set.

\section{The GRAND project}

GRAND will be the first stand-alone array of radio detectors measuring air showers spanning more than several $\mathrm{km}^{2}$. The largest effort to date, AERA [29], is only $17 \mathrm{~km}^{2}$ and is mostly triggered by the surface detector of the Pierre Auger Observatory. Using the radio technique over an area that is four orders of magnitude larger than this largest effort to date is a challenge that the GRAND collaboration confronts by a staged development 


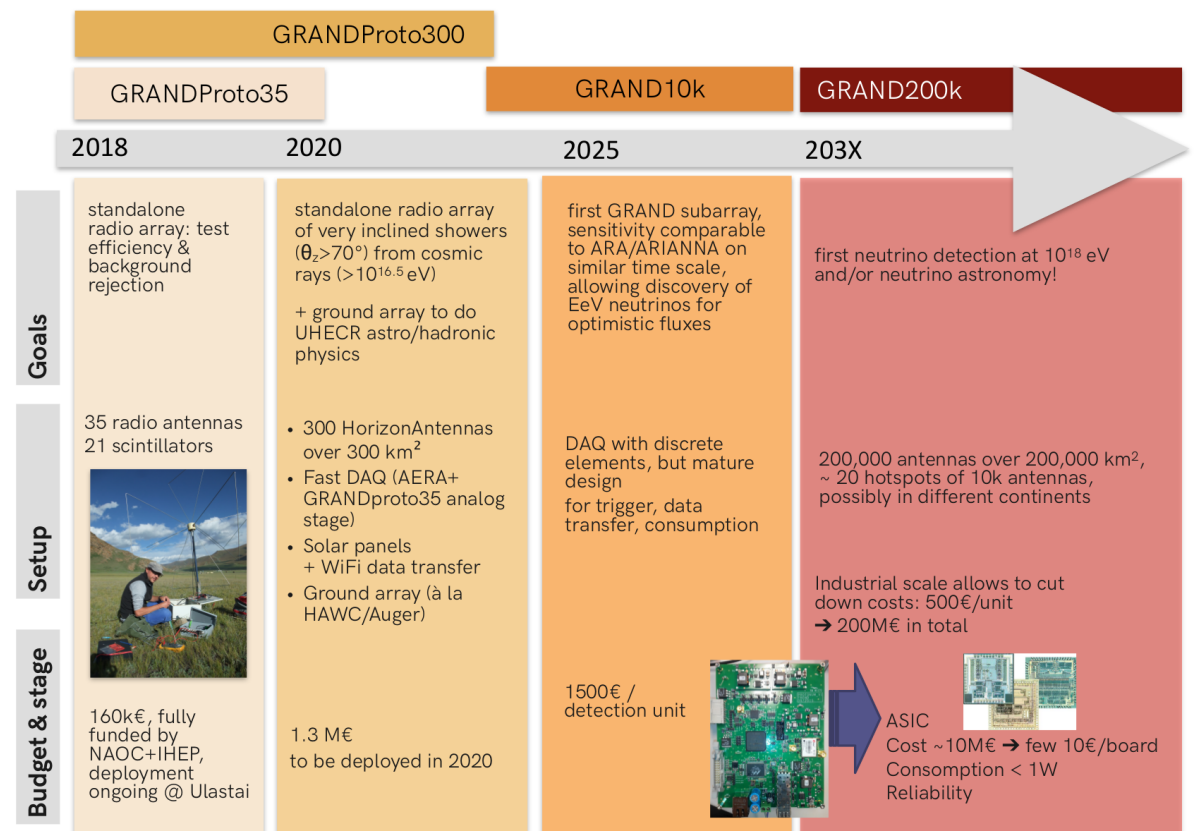

Figure 5: GRAND roadmap [1]

plan, shown in Figure 5. Each stage has its own goals and deliverables, both technical and scientific, and is described below.

\subsection{GRANDProto35}

GRANDProto35 is a combined array of 35 antennas and 21 scintillator detectors that is installed in the Tianshan mountains in China at the site of the 21CMA radio detector. The main purpose of this stage of GRAND is to verify the standalone trigger efficiency of radio detection of air showers as inferred from the TREND experiment [30] that was located at the same site. The TREND collaboration has performed a detailed study of the trigger efficiency and concluded that with a proper data acquisition system, trigger efficiencies of more than $80 \%$ can be obtained using the radio technique. GRANDProto35 will provide a more accurate efficiency measurement as the inefficiencies due to electronics and data acquisition, that were the main causes of event loss in TREND, are now negligible.

\subsection{GRANDProto300}

GRANDProto300 is being developed as a standalone radio array of 300 antennas on an area of more than $200 \mathrm{~km}^{2}$. The antenna spacing of the main part of the array will be $1 \mathrm{~km}$, which is the same as that foreseen for GRAND. The goal of this stage is to verify triggering on horizontal air showers, and develop the reconstruction for an array on a non-flat surface. In addition, the array will be used to test development of reliable and cheap stand-alone detection units, as well as different communication and data acquisition schemes.

It is foreseen that a particle detector array will also be installed in this area. For horizontal showers the ground array will mainly detect the muonic component of air showers, whereas the antenna array is sensitive to the electromagnetic part only. Combining these measurements will allow for a detailed study of the muon excess in high energy air showers as well as a comparison of the muon content and the depth of the shower maximum in the 
atmosphere as a function of the cosmic ray energy. This comparison provides the most accurate measurement of the composition of cosmic rays. The accessible energy region for this experiment is between 0.1 and $10 \mathrm{EeV}$, which allows the collaboration to study the transition region between galactic and extra-galactic cosmic rays.

\subsection{GRAND10K}

GRAND10K will be the first subarray of the GRAND detector. It will be deployed on an area of about $10,000 \mathrm{~km}^{2}$. Its neutrino sensitivity is comparable to that of future experiments like ARA [10] and ARIANNA [31 and it will be constructed in a similar timescale. The deployment, commissioning and initial running of GRAND10K will be used by the collaboration as the definite test for the whole detection chain before moving into a fully industrialised production mode that will be required for the construction of GRAND.

GRAND10K will be the first stage of GRAND were we expect to be sensitive to neutrino physics in case optimistic scenarios of neutrino production are realised in nature. In this stage of the experiment, the accuracy of the measurements combined with the expected statistics will surpass current current experiments, providing the collaboration with access to yet uncharted physics at the highest neutrino, photon and cosmic ray energies. In addition, the use of GRAND as a wide view radio telescope will provide information on the number of transients as well as their dispersion measure in a new frequency regime between 50 and $200 \mathrm{MHz}$.

\subsection{GRAND}

GRAND itself will consist of 10-20 subarrays. Each subarray will have to be located in a radio-quiet area, facing a mountain. This setup will allow for a full sky coverage of GRAND, provided its arrays are located in several parts of the world. In this way, GRAND will not only be sensitive to steady sources of ultra high energy neutrinos in all parts of the universe, but also its sensitivity to transient sources will be high most of the time. The coincidental high sensitivity of the Pierre Auger collaboration during the GW170817 event [32] illustrates the importance of such a distributed setup for the discovery of such events.

With GRAND, the collaboration will be able to access all of the physics outlined above and open the era of multi-messenger astro-particle physics at the highest energies. This is uncharted territory and is likely to provide surprises and new insights in the areas of astronomy and fundamental physics.

\section{Conclusion}

GRAND will be a multi-messenger astro-particle physics observatory sensitive to neutral and charged particles of the highest energies. The collaboration has developed a timeline for the development of GRAND that lasts for more than a decade. This time is needed to create affordable detection units as well as to ensure that design choices also work in the field. The stages defined in this process will allow for exciting new physics results at each stage. 


\section{References}

[1] J. Alvarez-Muniz et al. [GRAND Collaboration], arXiv:1810.09994 [astro-ph.HE].

[2] M. G. Aartsen et al. [IceCube Collaboration], Science 342 (2013) 1242856 doi:10.1126/science.1242856 [arXiv:1311.5238 [astro-ph.HE]].

[3] F. G. Schröder, arXiv:1811.01496 [astro-ph.IM].

[4] V. S. Berezinsky and G. T. Zatsepin, Phys. Lett. 28B (1969) 423. doi:10.1016/03702693(69)90341-4

[5] R. Alves Batista, R. M. de Almeida, B. Lago and K. Kotera, arXiv:1806.10879 [astroph.HE].

[6] M. G. Aartsen et al. [IceCube Collaboration], Astrophys. J. 809 (2015) no.1, 98 doi:10.1088/0004-637X/809/1/98 [arXiv:1507.03991 [astro-ph.HE]].

[7] M. G. Aartsen et al. [IceCube Collaboration], Phys. Rev. D 98 (2018) no.6, 062003 doi:10.1103/PhysRevD.98.062003 [arXiv:1807.01820 [astro-ph.HE]].

[8] A. Aab et al. [Pierre Auger Collaboration], Phys. Rev. D 91 (2015) no.9, 092008 doi:10.1103/PhysRevD.91.092008 [arXiv:1504.05397 [astro-ph.HE]].

[9] P. W. Gorham et al. [ANITA Collaboration], Phys. Rev. D 98 (2018) no.2, 022001 doi:10.1103/PhysRevD.98.022001 [arXiv:1803.02719 [astro-ph.HE]].

[10] P. Allison et al. [ARA Collaboration], Phys. Rev. D 93 (2016) no.8, 082003 doi:10.1103/PhysRevD.93.082003 [arXiv:1507.08991 [astro-ph.HE]].

[11] K. Murase, S. Inoue and S. Nagataki, Astrophys. J. 689 (2008) L105 doi:10.1086/595882 [arXiv:0805.0104 [astro-ph]].

[12] K. Fang and K. Murase, Phys. Lett. 14 (2018) 396 [Nature Phys. 14 (2018) no.4, 396] doi:10.1038/s41567-017-0025-4 [arXiv:1704.00015 [astro-ph.HE]].

[13] K. Fang, K. Kotera, K. Murase and A. V. Olinto, Phys. Rev. D 90 (2014) no.10, 103005 [Phys. Rev. D 90 (2014) 103005] Erratum: [Phys. Rev. D 92 (2015) no.12, 129901] doi:10.1103/PhysRevD.90.103005, 10.1103/PhysRevD.92.129901 [arXiv:1311.2044 [astro-ph.HE]].

[14] K. Murase, doi:10.1142/9789814759410_0002 arXiv:1511.01590 [astro-ph.HE].

[15] K. Murase and J. F. Beacom, Phys. Rev. D $81 \quad$ (2010) 123001 doi:10.1103/PhysRevD.81.123001 [arXiv:1003.4959 [astro-ph.HE]].

[16] B. Sarkar, K. H. Kampert and J. Kulbartz, doi:10.7529/ICRC2011/V02/1087

[17] A. Aab et al. [Pierre Auger Collaboration], JCAP 1704 (2017) no.04, 009 doi:10.1088/1475-7516/2017/04/009 [arXiv:1612.01517 [astro-ph.HE]].

[18] M. Niechciol [Pierre Auger Collaboration], PoS ICRC 2017 (2018) 517. doi:10.22323/1.301.0517

[19] G. Rubtsov et al. [Telescope Array Collaboration], PoS ICRC 2017 (2018) 551. doi:10.22323/1.301.0551 
[20] S. P. Knurenko and A. Sabourov, doi:10.5194/astra-7-251-2011 arXiv:1010.1185 [astro-ph.HE].

[21] T. Abu-Zayyad et al. [HiRes-MIA Collaboration], Astrophys. J. 557 (2001) 686 doi:10.1086/322240 [astro-ph/0010652].

[22] R. U. Abbasi et al. [HiRes Collaboration], Phys. Rev. Lett. 104 (2010) 161101 doi:10.1103/PhysRevLett.104.161101 [arXiv:0910.4184 [astro-ph.HE]].

[23] J. Belz [Telescope Array Collaboration], PoS ICRC 2015 (2016) 351. doi:10.22323/1.236.0351

[24] J. Bellido [Pierre Auger Collaboration], PoS ICRC 2017 (2018) 506. doi:10.22323/1.301.0506

[25] P. Sanchez-Lucas [Pierre Auger Collaboration], PoS ICRC 2017 (2018) 495. doi:10.22323/1.301.0495

[26] S. Buitink et al., Nature 531 (2016) 70 doi:10.1038/nature16976 [arXiv:1603.01594 [astro-ph.HE]].

[27] P. Homola, M. Risse, D. Gora, D. Heck, H. Klages, J. Pekala, B. Wilczynska and H. Wilczynski, Nucl. Phys. Proc. Suppl. 151 (2006) 119. doi:10.1016/j.nuclphysbps.2005.07.022

[28] H. Schoorlemmer, PhD thesis, University of Nijmegen, 2008, ISBN: 978-90-9027039-5

[29] A. Aab et al. [Pierre Auger Collaboration], JCAP 1810 (2018) no.10, 026 doi:10.1088/1475-7516/2018/10/026 [arXiv:1806.05386 [astro-ph.IM]].

[30] D. Charrier et al., arXiv:1810.03070 [astro-ph.HE].

[31] C. Persichilli [ARIANNA Collaboration], PoS $\quad$ ICRC $2017 \quad$ (2018) 977. doi:10.22323/1.301.0977

[32] A. Albert et al. [ANTARES and IceCube and Pierre Auger and LIGO Scientific and Virgo Collaborations], Astrophys. J. 850 (2017) no.2, L35 doi:10.3847/20418213/aa9aed [arXiv:1710.05839 [astro-ph.HE]]. 